\title{
COHOMOLOGY OF DEFORMATIONS
}

\author{
URI BADER AND PIOTR W. NOWAK
}

\begin{abstract}
In this article we study cohomology of a group with coefficients in representations on Banach spaces and its stability under deformations. We show that small, metric deformations of the representation preserve vanishing of cohomology. As applications we obtain deformation theorems for fixed point properties on Banach spaces. In particular, our results yield fixed point theorems for affine actions in which the linear part is not uniformly bounded. Our proofs are effective and allow for quantitative estimates.
\end{abstract}

Cohomology with coefficients in representations on Banach spaces is a broad topic, encompassing important notions like fixed point properties and Kazhdan's property (T), amenability, a-T-menability or the Haagerup property, and their generalizations, as well as $\ell_{p}$-cohomology. The main theme in this paper is how does cohomology of a group with coefficients in a representation on a Banach space change under small, metric perturbations of that representation. Let $\Gamma$ be a group generated by a finite set $S$. Given a representation of $\Gamma$ on a Banach space $E$ and $\varepsilon>0$, a representation $\rho$ of $\Gamma$ on the same Banach space $E$ is said to be an $\varepsilon$-deformation of $\pi$ if

$$
\sup _{s \in S}\left\|\pi_{s}-\rho_{s}\right\|_{B(E)} \leq \varepsilon .
$$

We are interested in the stability of the behavior of cohomology groups under such deformations of representations. In degree $1, \pi$-cocycles correspond to affine actions with linear part $\pi$ and in this case the question we are interested in is whether for an $\varepsilon$-deformation $\rho$ of $\pi$ as above, properties of the affine $\pi$-actions influence the properties of affine $\rho$-actions. The main result we prove is a deformation principle for vanishing of cohomology with coefficients in Banach modules.

Theorem 1. Let $\Gamma$ be a group of type $\mathrm{F}_{n+1}, n \geq 1$, with a corresponding Eilenberg-MacLane space $X$ and let $\pi$ be a representation of $\Gamma$ on a Banach space $E$. Assume that

(1) $H^{n}(\Gamma, \pi)=0$,

(2) $H^{n+1}(\Gamma, \pi)$ is reduced.

Then there exists a constant $\varepsilon=\varepsilon(\Gamma, X, \pi, n)>0$, such that for every $\varepsilon$-deformation $\rho$ of $\pi$ we have

$$
H^{n}(\Gamma, \rho)=0 .
$$

The first author was partially supported by the European Research Council. The second author was partially supported by the Foundation for Polish Science. 
Additionally, the $n$-th Kazhdan constant can be estimated explicitly.

Theorem 1 will follow from a more general version in which we consider the $L_{p}$-cohomology with coefficients twisted by $\pi$ for $1 \leq p<\infty$.

Theorem 2 (Theorem $1^{\prime}$ ). Let $\Gamma$ be a group acting on a uniformly locally finite simplicial complex $X, \pi$ be a representation of $\Gamma$ on a Banach space $E$ and let $1 \leq p<\infty$. Assume that

(1) $L_{p} H^{n}(X, \Gamma, \pi)=0$,

(2) $L_{p} H^{n+1}(X, \Gamma, \pi)$ is reduced.

Then there exists a constant $\varepsilon=\varepsilon(\Gamma, X, \pi, n, p)>0$, such that for every $\varepsilon$-deformation $\rho$ of $\pi$ we have

$$
L_{p} H^{n}(X, \Gamma, \rho)=0 .
$$

Note that we do not assume any uniform boundedness of either representation. However, even if $\pi$ is an isometric representation, an $\varepsilon$-deformation $\rho$ does not have to be uniformly bounded - all that matters is the distance between the generators. Natural examples of such transformations can be obtained by twisting a given representation by an appropriately chosen cocycle. We discuss such examples in Section 1.

In 4.4 we also discuss an example showing that the second assumption that the cohomology group $H^{n+1}(\Gamma, \pi)$ is reduced cannot be dropped. As a byproduct of our methods we also obtain a deformation principle for cocycles: if $\rho$ is an $\varepsilon$-deformation of $\pi$ for a sufficiently small $\varepsilon>0$, then the cocycles for $\rho$ lie close, in an appropriate sense, to cocycles for $\pi$, provided that $H^{n+1}(\Gamma, \pi)$ (or $L_{p} H^{n+1}(\Gamma, \pi)$ ) is reduced. See Theorem 26 in the text for the precise formulation.

There are many instances in which the condition that $H^{n+1}(\Gamma, \pi)$ is reduced is satisfied. One example is when the representation $\pi$ is finite-dimensional, then automatically, $H^{n+1}(\Gamma, \pi)$ is reduced. This is clear in the case when $\Gamma$ is of type $F_{n+1}$, see also [1] for the general case. Another natural case is when $H^{n+1}$ vanishes. Examples of vanishing theorems for higher-dimensional cohomology with coefficients in unitary representations can be found in $[6,9]$ and include "vanishing up to the rank" phenomena and vanishing for automorphism groups of thick buildings. Interestingly, the class of groups for which the assumptions of Theorem 1 are always satisfied is related to higherdimensional analogs of Kazhdan's property (T). We discuss this phenomenon in Section 5.1.

The higher Kazhdan constants, mentioned in the above theorems, are defined in section 2.3. They are higher dimensional analogs of the usual Kazhdan constant for groups with property (T). We define the $n$-th Kazhdan constant in the setting of cohomology, under the assumption that the cohomology in degree $n+1$ is reduced.

The main application and the original motivation for Theorem 1 are fixed point properties for actions of discrete groups on Banach spaces. In degree 1 , the vanishing of cohomology of $\Gamma$ with coefficients in a representation $\pi$ 
translates to a fixed point property for affine actions of $\Gamma$ with linear part $\pi$. A classical example is Kazhdan's property (T), which for a group $\Gamma$ was characterized by Delorme and Guichardet by vanishing of cohomology in degree 1 with coefficients in every unitary representation of $\Gamma$, see [5]. Vanishing of cohomology with coefficients in representations on more general Banach spaces was studied and discussed in, e.g. $[2,3,11,16,18,19]$. Thus in degree 1 Theorem 1 yields a deformation result for actions with fixed points for finitely presented groups.

Theorem 3. Let $\Gamma$ be finitely presented with a finite generating set $S$. Let $H^{2}(\Gamma, \pi)$ be reduced and assume that every affine action of $\Gamma$ with linear part $\pi$ has a fixed point. Then there exists $\varepsilon=\varepsilon(\Gamma, S, \pi)>0$ such that if $\rho$ is an $\varepsilon$ deformation of $\pi$ then every affine action with linear part $\rho$ has a fixed point.

Here, similarly as before, we can replace finite presentability by merely finite generation if we assume that $L_{p} H^{2}(\Gamma, \pi)$ is reduced, instead of $H^{2}(\Gamma, \pi)$. Since Kazhdan's property (T) is characterized by the existence of a fixed point for every affine isometric action, we obtain the following

Theorem 4. Let $\Gamma$ be finitely presented with a finite generating set $S$. If $\Gamma$ has property $(T)$ then for every unitary representation $\pi$ such that $H^{2}(\Gamma, \pi)$ is reduced there exists $\varepsilon=\varepsilon(\Gamma, S, \pi)>0$ such that for every $\varepsilon$-deformation $\rho$ of $\pi$, every affine isometric action with linear part $\rho$ has a fixed point.

If $H^{2}(\Gamma, \pi)$ is reduced for every unitary representation $\pi$ then the $\varepsilon$ above can be chosen independently of $\pi$.

Theorem 4 applies to automorphism groups of thick buildings, which exhibit vanishing of higher cohomology with coefficients in any unitary representation under appropriate link conditions $[4,9,17]$.

The above corollaries of Theorem 1 can be compared with the results of Fisher and Margulis [11]. A map $\varphi: X \rightarrow X$ of a metric space $X$ is an $\varepsilon$ isometry if it satisfies the bi-Lipschitz condition

$$
(1-\varepsilon)\|v-w\| \leq\|\varphi(x)-\varphi(y)\| \leq(1+\varepsilon)\|v-w\| .
$$

Theorem 1.6 in [11] states that for a locally compact, $\sigma$-compact group $\Gamma$ with property (T) and compact generating set $K$, there exists $\varepsilon>0$, depending on $\Gamma$ and $K$ only, such that for any continuous action of $\Gamma$ on a Hilbert space, where $K$ acts by $\varepsilon$-almost isometries, there exists a fixed point.

Note that for a finitely generated group, if a representation $\rho$ is an $\varepsilon$ deformation of a unitary representation, then the generating set acts by $\varepsilon$ isometries. Thus our theorem in this case gives a statement similar to the one proved by Fisher and Margulis. However, our methods have several advantages. First, the proof in [11] is indirect and does not give any insight into the value of $\varepsilon$ for which the theorem holds. Our Theorem 1 not only extends this type of phenomena to higher cohomology, but also gives explicit estimates of the $\varepsilon$ in terms of higher Kazhdan constants. Indeed, our arguments are effective and the behavior of various constants can be traced throughout the 
proofs. Second, the argument used in [11] is not available in the same generality as Theorem 1. Indeed, the argument in [11] is based on an ultrapower construction and relies on the fact that the ultrapower of a Hilbert space is again a Hilbert space. A similar fact for $L_{p}$-spaces was proved by Heinrich and Mankiewicz [13], however for general Banach spaces such methods are not available.

An important feature of Theorem 1 is that we obtain information about higher cohomology groups. In particular, we can deform the top dimensional cohomology of a group of geometric dimension $n$. Then we can conclude that deformations in dimension $n$ preserve the vanishing of cohomology.

Corollary 5. Let $\Gamma$ be a group of geometric dimension $n$ and a corresponding Eilenberg-MacLane space $X$. If $H^{n}(\Gamma, \pi)=0$ then there exists $\varepsilon=\varepsilon(\Gamma, X, \pi)>0$ such that

$$
H^{n}(\Gamma, \rho)=0
$$

for any $\varepsilon$-deformation $\rho$ of $\pi$.

Another situation, mentioned earlier, in which $H^{n+1}(\Gamma, \pi)$ is automatically reduced is when $\Gamma$ is of type $F_{n+1}$ and $\pi$ is finite-dimensional. In that case we obtain the following

Corollary 6. Let $\Gamma$ be of type $F_{n+1}$ with a corresponding Eilenberg-MacLane space $X$ and let $\pi$ be a finite-dimensional representation of $\Gamma$. If $H^{n}(\Gamma, \pi)=0$ then there exists $\varepsilon=\varepsilon(\Gamma, X, \pi)>0$ such that

$$
H^{n}(\Gamma, \rho)=0
$$

for every $\varepsilon$-deformation $\rho$ of $\pi$.

The last application that we derive is the following criterion for non-vanishing of cohomology.

Corollary 7. Let $\Gamma$ be of type $F_{n+1}$ and let $\pi$ be a representation such that $H^{n}(\Gamma, \pi)=0$ and there exists a family $\pi_{i}$ of deformations converging to $\pi$ such that $H^{n}\left(\Gamma, \rho_{i}\right) \neq 0$. Then $H^{n+1}(\Gamma, \pi) \neq 0$.

We stress that all the arguments are effective and quantitative, and it is possible to estimate all the constants. We do not to compute the exact estimates and instead we indicate what do they depend on.

Acknowledgements. We would like to thank Roman Sauer and Shmuel Weinberger for helpful comments. We are also grateful to Narutaka Ozawa for bringing [8] to our attention.

\section{CONTENTS}

Acknowledgements

1. Deformations of representations

1.1. The space $\operatorname{Hom}\left(\Gamma, B_{i n v}(E)\right)$

1.2. Deformations 
2. Cohomology 7

2.1. Finiteness conditions 7

2.2. Cohomology with coefficients in a representation 7

2.3. Higher Kazhdan constants 9

3. Geometry of subspaces and quotients $\quad 10$

4. Cohomology of deformations 14

4.1. Deformations of cocycles $\quad 15$

4.2. Proof of the main theorem $\quad 15$

4.3. Proof in the Hilbert case $\quad 17$

4.4. Assumptions on $H^{n+1}$ are essential 18

$\begin{array}{ll}\text { 5. Final remarks } & 19\end{array}$

5.1. Higher property (T) and reduced cohomology 19

5.2. Weil's local rigidity criterion 20

5.3. Vanishing vs connectedness of $\operatorname{Hom}(\Gamma, U(\not{H}))$

$\begin{array}{ll}\text { References } & 22\end{array}$

\section{DeFormations of REPRESENTATIONS}

1.1. The space $\operatorname{Hom}\left(\Gamma, B_{i n v}(E)\right)$. Consider a finitely generated group $\Gamma$ with a fixed finite generating set $S=S^{-1}$. Let $E$ be a Banach space and denote by $B_{i n v}(E)$ the set of linear self-isomorphisms of $E$, equipped with the operator norm.

Given a subset $W \subseteq B_{i n v}(E)$ by $\operatorname{Hom}(\Gamma, W)$ we will denote the space of homomorphisms $\varphi: \Gamma \rightarrow W$ (depending on $W$ it can happen that $\operatorname{Hom}(\Gamma, W)$ is empty). We equip $\operatorname{Hom}(\Gamma, W)$ with the metric

$$
d_{S}(\pi, \rho)=\sup _{s \in S}\|\pi(s)-\rho(s)\|,
$$

and the corresponding uniform topology.

In particular, if Iso $(E)$ denotes the group of isometries of a Banach space $E$, the space $\operatorname{Hom}(\Gamma, \operatorname{Iso}(E))$ is the space of isometric representations of $\Gamma$ on $E$.

\subsection{Deformations.}

Definition 8. Let $\Gamma$ be a group generated by a finite set $S$. Given a representation $\pi$ of $\Gamma$ on a Banach space $E$ and $\varepsilon>0$, a representation $\rho$ of $\Gamma$ on $E$ is said to be an $\varepsilon$-deformation of $\pi$ if

$$
d_{S}(\pi, \rho) \leq \varepsilon .
$$

The most basic example of a deformation can be obtained by defining

$$
\rho_{\gamma}=T \pi_{\gamma} T^{-1},
$$

where $T$ is an isomorphism of the Banach space on which $\pi$ is defined, with $\|I-T\| \leq \varepsilon$ appropriately small. However, in this case $T$ also induces an 
isomorphism of cohomology groups $H^{*}(\Gamma, \pi)$ and $H^{*}(\Gamma, \rho)$. Thus Theorem 1 for such deformations is trivially true and they are not interesting for us.

In the case $\Gamma=\mathbb{F}_{n}$ it is particularly easy to construct deformations. Indeed, if $S$ is the free generating set then any map $S \rightarrow B_{\text {inv }}(H)$ extends to a representation of $\mathbb{F}_{n}$. Any two such maps that are within $d_{S}$ distance $\varepsilon$, define representations which are deformations of each other.

Given a group $\Gamma$ with a $H$ and an appropriate representation of $H$ one can construct the induced representation of $\Gamma$. Using this idea in [8, Section 4] the authors constructed a family of pairwise non-equivalent deformations of the regular representation of any group with a free subgroup.

Natural examples of nontrivial deformations for other groups can also be obtained by twisting a representation by an appropriately chosen non-trivial cocycle, which is small in norm on the generators. Below we give examples of such deformations.

Example 9. Let $\Gamma$ act on measure space $(\Omega, \mu)$, with measure $\mu$ finite, by measure class preserving transformations. The action of $\Gamma$ induces a unitary representation $\pi$ on $L_{2}(\Omega, \mu)$,

$$
\pi_{g} f=(g \cdot f)\left(\frac{d g \mu}{d \mu}\right)^{1 / 2} .
$$

If the action of $\Gamma$ does not preserve the measure then

$$
\rho_{g} f=(g \cdot f)\left(\frac{d g \mu}{d \mu}\right)^{\alpha}
$$

where $\alpha \in(0, \infty)$, defines a family of deformations of $\pi$.

Example 10. Let $\pi, \pi^{\prime}$ be representations on a Banach space $E$ and consider the collection of operators

$$
T_{g}=\left(\begin{array}{cc}
\pi_{g} & D_{g} \\
0 & \pi_{g}^{\prime}
\end{array}\right)
$$

on $E \oplus E$. It is easy to check that the $T_{g}$ form a representation of $\Gamma$ if and only if $D$ is a derivation $D: \Gamma \rightarrow B(E)$, where $B(E)$ is considered to be a $\Gamma$ bimodule by composing with $\pi$ on the left and $\pi^{\prime}$ on the right; that is, $D$ satisfies $D(g h)=\pi_{g} D(h)+D(g) \pi_{h}^{\prime}$. Given such a derivation $D: \Gamma \rightarrow B(E)$ we define

$$
\rho_{g}^{(\alpha)}=\left(\begin{array}{cc}
\pi_{g} & \alpha D_{g} \\
0 & \pi_{g}^{\prime}
\end{array}\right)
$$

where $\alpha \in[0, \infty)$. It is easy to see that this gives a family of deformations of the representation

$$
\rho_{g}^{(0)}=\left(\begin{array}{cc}
\pi_{g} & 0 \\
0 & \pi_{g}^{\prime}
\end{array}\right)
$$

A representation $\pi$ on a Hilbert space $E$ is unitarizable if it is similar to a unitary representation. An obvious necessary condition for a representation to be unitarizable is that $\sup _{\gamma \in \Gamma}\left\|\pi_{\gamma}\right\|<\infty$. Note that the deformations of unitary representation discussed in Examples 9 and 10 do not have to be 
uniformly bounded. In the case of Example 10 it is known (see [20]) that $\rho^{(\alpha)}$ is unitarizable if and only if the derivation $D$ is inner; that is, there exists $T \in B(H)$ such that $D_{\gamma}=\pi_{\gamma} T-T \pi_{\gamma}^{\prime}$ for every $\gamma \in \Gamma$.

\section{Cohomology}

The general reference on cohomology of groups is [7]. Let $\Gamma$ act on $X$, which is a uniformly locally finite simplicial complex, by simplicial automorphisms. In $X$ there is a fundamental domain $F \subseteq X$, which we assume to be a subcomplex.

2.1. Finiteness conditions. Recall that a group $\Gamma$ is said to be of type $\mathrm{F}_{n}$ if it admits an Eilenberg-Maclane space $B \Gamma$ which is a simplicial complex with a finite $n$-skeleton. $\Gamma$ is said to be of type $\mathrm{F}_{\infty}$ if it is of type $\mathrm{F}_{n}$ for every $n \geq 1$. See $[7,12]$ for a discussion. Such finiteness properties will be important in our considerations. The condition $\mathrm{F}_{1}$ is equivalent to the group $\Gamma$ being finitely generated, while $\mathrm{F}_{2}$ holds if and only if the group $\Gamma$ is finitely presented. Examples of groups satisfying such finiteness conditions include e.g. hyperbolic groups, combable groups, Thompson groups, $\mathrm{SL}_{n}(\mathbb{Z})$.

In the case when the group $\Gamma$ is of type $\mathrm{F}_{n}$, we can choose $X$ to be contractible with the fundamental domain $F$ above having finite skeletons $F^{(k)}$ for $k=0,1, \ldots, n$, and with $\Gamma$ acting by simplicial automorphisms. Let

$$
S=\{g \in \Gamma: \bar{F} \cap g \cdot \bar{F} \neq \varnothing\} \subseteq \Gamma .
$$

Under the condition $\mathrm{F}_{1}$ we have that $S$ is finite and $S$ generates $\Gamma$. Our convention is that whenever we are considering a group $\Gamma$ of type $F_{n}$, then there is a chosen Eilenberg-MacLane space $X$ of $\Gamma$ witnessing this requirement; i.e., $X$ is a simplicial complex with a finite $n$-skeleton.

2.2. Cohomology with coefficients in a representation. Let $X$ be a locally finite simplicial complex with a simplicial, free action of $\Gamma$ and let $F \subset X$ be a fundamental domain. Let $\pi$ be a representation of $\Gamma$ on a Banach space $E$. For a simplex $\sigma \in X$ we denote by $\gamma_{\sigma} \in \Gamma$ the unique element $\gamma \in \Gamma$ such that $\gamma_{\sigma} \cdot \sigma \in F$. As usual, let $X^{(n)}$ denote the $n$-skeleton of a complex $X$; that is, the collection of all $n$-simplices of $X$.

For $1 \leq p<\infty$ the space of $p$-integrable (untwisted) cochains is the space

$C_{(p)}^{n}(X, \Gamma, E)=\left\{f: X^{(n)} \rightarrow E: f\right.$ alternating, $\left.f(\sigma)=f\left(\gamma_{\sigma} \cdot \sigma\right), \sum_{\sigma \in F^{(n)}}\|f(\sigma)\|_{E}^{p}<\infty\right\}$.

For a simplex $\sigma=\left(v_{0}, \ldots, v_{k}\right)$ denote $\sigma_{i}=\left(v_{0}, \ldots, \hat{v}_{i}, \ldots, v_{k}\right)$. The codifferential is the map $d_{\pi}^{n}: C_{(p)}^{n}(X, \Gamma, E) \rightarrow C_{(p)}^{n+1}(X, \Gamma, E)$ given by the formula

$$
d_{\pi}^{n} f(\sigma)=\sum_{i=0}^{n}(-1)^{i} \pi_{g_{\sigma}^{-1}} \pi_{g_{\sigma_{i}}} f\left(\sigma_{i}\right)
$$

If $\Gamma$ is of type $\mathrm{F}_{k}$ the space of cochains, $C_{(p)}^{n}(X, \Gamma, E)$, admits a Banach space structure for every $n \leq k$. Indeed, the $n$-cochains $C_{(p)}^{n}(X, \Gamma, E)$ can be viewed 
as a subspace of the direct sum over the simplices of $F^{(n)}$, with a natural Banach space structure via the norm

$$
\|f\|_{p}=\left(\sum_{\sigma \in F^{(n)}}\|f(\sigma)\|_{E}^{p}\right)^{1 / p} .
$$

The dual space $\left(C_{(p)}^{n}(X, \Gamma, E)\right)^{*}$ is then isometrically isometric with a quotient $C_{(q)}^{n}\left(X, \Gamma, E^{*}\right)$ of this direct sum, equipped with the norm $\|\cdot\|_{q}$, where $p^{-1}+$ $q^{-1}=1$.

The codiferrentials are bounded linear operators for every $1 \leq p<\infty$ with respect to the above norms, see e.g., [4,14].

The $L_{p}$-cohomology groups of $X$ with coefficients twisted by $\pi$ are defined as the cohomology of the above cochain complex:

$$
L_{p} H^{n}(X, \Gamma, \pi)=\operatorname{ker} d_{\pi}^{n} / \operatorname{im} d_{\pi}^{n-1} .
$$

We can also consider the reduced cohomology,

$$
L_{p} \bar{H}^{n}(X, \Gamma, \pi)=\operatorname{ker} d_{\pi}^{n} / \overline{\operatorname{im} d_{\pi}^{n-1}},
$$

where $\overline{\operatorname{im} d_{\pi}^{n-1}}$ denotes the norm closure of the image of $d_{\pi}^{n-1}$. We say that $L_{p} H^{n}(X, \Gamma, \pi)$ is reduced if $L_{p} \bar{H}^{n}(X, \Gamma, \pi)=L_{p} H^{n}(X, \Gamma, \pi)$.

Remark 11. The above definition of $L_{p}$-cohomology with coefficients in the $\Gamma$-module $(E, \pi)$ agrees with the one considered earlier in $[4,14]$. Usually one considers twisted cochains $C^{n}(X, \pi)$, satisfying $f(\gamma \cdot \sigma)=\pi(g) f(\sigma)$ with the standard differential, $d^{n} f(\sigma)=\sum_{i=0}^{n}(-1)^{i} f\left(\sigma_{i}\right)$. We then define the "untwisting map" $\varphi^{n}: C^{n}(X, \Gamma, \pi) \rightarrow C^{n}(X, \Gamma, E)$ by

$$
\varphi^{n}(f)(\sigma)=\pi\left(g_{\sigma}^{-1}\right) f(\sigma)=f\left(g_{\sigma}^{-1} \cdot \sigma\right) .
$$

One can then check, that

$$
d_{\pi}^{n} \circ \varphi^{n+1}=\varphi^{n} \circ d^{n-1} .
$$

Remark 12. In the case when $X$ is uniformly locally finite we can also consider the $\ell_{\infty}$-cochains,

$C_{(\infty)}^{n}(X, \Gamma, E)=\left\{f: X^{(n)} \rightarrow E: f\right.$ alternating, $\left.f(\sigma)=f\left(\gamma_{\sigma} \cdot \sigma\right), \sup _{\sigma \in X^{(n)}}\|f(\sigma)\|_{E}<\infty\right\}$.

Under the assumption of uniform local finiteness of $X$ we have that the codifferential is a bounded linear operator.

In the case when $X$ is contractible and the action of $\Gamma$ on $X$ is cocompact,

$$
L_{p} H^{n}(X, \Gamma, \pi)=H^{n}(\Gamma, \pi),
$$

is the group cohomology of $\Gamma$ with coefficients in the $\Gamma$-module $(E, \pi)$.

We have the following interpretation of the vanishing of $L_{p} H^{n}(X, \Gamma, \pi)$. The fact that $L_{p} H^{n}(X, \Gamma, \pi)=0$ is, by definition, the surjectivity of the codifferential

$$
d_{\pi}^{n-1}: C_{(p)}^{n-1}(X, \Gamma, E) \rightarrow \operatorname{ker} d_{\pi}^{n} .
$$


Consider the adjoint map

$$
\left(d_{\pi}^{n-1}\right)^{*}:\left(\operatorname{ker} d_{\pi}^{n}\right)^{*} \rightarrow C_{(p)}^{n-1}(X, \Gamma, E) .
$$

Standard duality arguments yield the following

Proposition 13. Let $\Gamma$ be a group acting on a uniformly locally finite simplicial complex $X$ and let $\pi$ be a representation of $\Gamma$ on a Banach space $E$. The following conditions are equivalent.

(1) $L_{p} H^{n}(X, \Gamma, \pi)=0$,

(2) There exists a constant $C>0$ such that

$$
\left\|\left(d_{\pi}^{n-1}\right)^{*} \varphi\right\| \geq C\|\varphi\|
$$

for every $\varphi \in\left(\operatorname{ker} d_{\pi}^{n}\right)^{*}$.

The above proposition will be one of our main tools in the proof of Theorem 1. A similar strategy was used in [18].

2.3. Higher Kazhdan constants. Recall that for a group $\Gamma$, generated by a finite set $S$, the Kazhdan constant is the number

$K(\Gamma, S)=\inf \left\{\sup _{s \in S} \frac{\left\|\pi_{s} v-v\right\|}{\|v\|}: \pi\right.$ unitary, with no non-zero invariant vectors $\}$.

A group $\Gamma$ has Kazhdan's property (T) if and only if $K(\Gamma, S)>0$. We will define a higher Kazhdan-type constant in the context of cohomology.

Definition 14. Let $\Gamma$ be a group acting on a uniformly locally finite simplicial complex $X$ and let $\pi$ be a representation of $\Gamma$ on a Banach space $E$. If the cohomology $L_{p} H^{n+1}(X, \Gamma, \pi)$ is reduced then the number

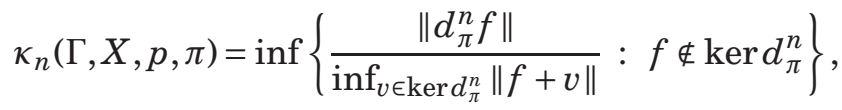

will be called the $n^{\text {th }}$-Kazhdan constant of the triple $(\Gamma, X, \pi)$.

Another way to define the constant $\kappa_{n}(\Gamma, X, p, \pi)$ is as follows. Since the image of $d_{\pi}^{n}$ is closed in $C_{(p)}^{n+1}(X, \Gamma, E)$, the map $d_{\pi}^{n}$ descends to an isomorphism $\widetilde{d}_{\pi}^{n}$ between $C_{(p)}^{n}(X, \Gamma, E) / \operatorname{ker} d_{\pi}^{n}$ and $\operatorname{im} d_{\pi}^{n} \subseteq C_{(p)}^{n+1}(X, \Gamma, E)$.

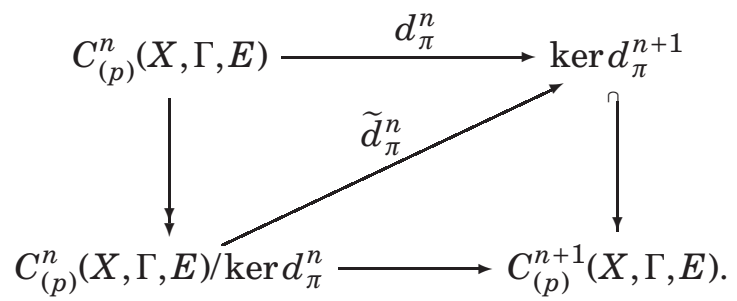

The constant $\kappa_{n}(\Gamma, X, p, \pi)$ is then the supremum of those constants $D>0$ that satisfy

$$
\left\|\widetilde{d}_{\pi}^{n} v\right\| \geq D\|v\|,
$$

for every $v \in C_{(p)}^{n}(X, \Gamma, E) / \operatorname{ker} d_{\pi}^{n}$. 
Let now $\mathscr{P}$ be any family of representations of $\Gamma$ on a Banach spaces that is closed under taking infinite direct sums. For instance, unitary representations, or more generally, uniformly bounded representations on a Hilbert space, whose norms are all bounded by a uniform constant, form such families. On the other hand, finite-dimensional representations do not form such a family.

Proposition 15. Let $\Gamma$ act on a uniformly locally finite simplicial complex $X$. Let $n \geq 1$ and let $\mathscr{P}$ be as above. If $L_{p} H^{n}(X, \Gamma, \pi)$ is reduced for every representation $\pi \in \mathscr{P}$ then the $n$-th Kazhdan constant of $\mathscr{P}$

$$
\kappa_{n}(\Gamma, X, p, \mathscr{P})=\inf \{\kappa(\Gamma, X, p, \pi): \pi \in \mathscr{P}\}>0 .
$$

Proof. From the condition $L_{p} H^{n}(X, \Gamma, \pi)$ is reduced we have $\kappa(\Gamma, X, p, \pi)>0$ for every $\pi \in \mathscr{P}$. Assume the contrary and let $\pi_{n}$ be a sequence of representations in $\mathscr{P}$, such that $\kappa\left(\Gamma, X, p, \pi_{n}\right) \rightarrow 0$. Let $\pi=\oplus_{n \in \mathbb{N}} \pi_{n}$ be a representation on $\bigoplus_{n \in \mathbb{N}} \mathscr{H}$. By the assumption on $\mathscr{P}, \pi \in \mathscr{P}$. Then

$$
\kappa_{n}(\Gamma, X, p, \mathscr{P}) \leq \kappa_{n}\left(\Gamma, X, p, \pi_{n}\right),
$$

for every $n \in \mathbb{N}$.

For instance, if $\mathscr{P}$ is the class of unitary representations of a finitely presented $\Gamma$, then $\kappa_{0}(\Gamma, X, \infty, \mathscr{P})$ is essentially the Kazhdan constant of $\Gamma$ (for the generating set determined by the action of $\Gamma$ on $X$ ). If $\mathscr{P}$ is the class of unitary representations factoring through finite quotients then $\kappa_{n}(\Gamma, X, \infty, \mathscr{P})>0$ if and only if $\Gamma$ has property $(\tau)[15]$.

\section{Geometry of Subspaces AND QUotients}

In order to analyze the cohomology with coefficient in a deformation we will need to look closely at the geometry of the spaces of cocycles and discuss a general setting for comparing norm bounds on operators on such spaces. The following notion of close subspaces was studied in [9] and used later in [10] in the context of Hilbert spaces. Below we will consider this notion for subspaces of Banach spaces.

Definition 16. Let $E$ be a Banach space and $V, W$ be two subspaces of $E$. Let $\varepsilon>0$. We say that $V$ is $\varepsilon$-close to $W$ if for every $v \in V$ there exists $w \in W$ such that

$$
\|v-w\| \leq \varepsilon\|v\|
$$

We will not, in general, assume that $V$ and $W$ are closed. If $E=\mathscr{H}$ is a Hilbert space then, if the subspace $W$ is closed in $\mathscr{H}$, in the above definition we can replace $w$ by $P_{W} v$, where $P_{W}: \not{H} \rightarrow W$ is the orthogonal projection onto $W$. In general, $w$ can be taken to be the (non-linear) nearest point projection.

Lemma 17. Let $V$ and $W$ be two closed subspaces of a Hilbert space $\not{H}$.

(1) If $V$ is $\varepsilon$-close to $W$ then $\left\|P_{W} v\right\| \geq(1-\varepsilon)\|v\|$ for every $v \in V$;

(2) if $\left\|P_{W} v\right\| \geq c\|v\|$ for every $v \in V$ then $V$ is $\sqrt{1-c}$-close to $W$. 
Proof. The first claim follows after applying the triangle inequality

$$
\|v\|-\left\|P_{W} v\right\| \leq\left\|v-P_{W} v\right\| \leq \varepsilon\|v\| .
$$

The second inequality is a consequence of the estimate

$$
\left\|v-P_{W} v\right\|^{2}=\|v\|^{2}-\left\|P_{W} v\right\|^{2} \leq(1-c)\|v\|^{2} .
$$

In the case of closed subspaces of Hilbert spaces a more detailed discussion on the $\varepsilon$-closeness and a related notion of $\varepsilon$-orthogonality can be found in [10].

The following lemmas describe the behavior of bounded linear operators on close subspaces.

Lemma 18. Assume that $V, W$ are subspaces of a Banach space $E$. If $V$ is $\varepsilon$-close to $W$ then

(1) if $W \subseteq Y$ then $V$ is $\varepsilon$-close to $Y$; in particular, $V$ is $\varepsilon$-close to $\bar{W}$;

(2) $\bar{V}$ is $\varepsilon^{\prime}$-close to $W$ for every $\varepsilon^{\prime}>\varepsilon$.

Proof. This is obvious.

Lemma 19. Let $E, E^{\prime}$ be Banach spaces and $V \subseteq E$ be a subspace. Let $T, S$ : $E \rightarrow E^{\prime}$ be linear bounded operators such that $\|T-S\| \leq \varepsilon$. If $\|T v\| \geq C\|v\|$ for every $v \in V$ then

$$
\|S v\| \geq(C-\varepsilon)\|v\|
$$

for every $v \in V$.

Proof. We have

$$
\begin{aligned}
\|S v\| & =\|S v-T v+T v\| \\
& \geq\|T v\|-\|(T-S) v\| \\
& \geq C\|v\|-\varepsilon\|v\| .
\end{aligned}
$$

Lemma 20. Let $E, E^{\prime}$ be Banach spaces, $V, W$ be closed subspaces of $E$. Let $T: E \rightarrow E^{\prime}$ be a bounded linear operator. If $\|T w\| \geq C\|w\|$ for every $w \in W$ and $V$ is $\varepsilon$-close to $W$ then

$$
\|T v\| \geq(C-\varepsilon-\varepsilon\|T\|)\|v\|,
$$

for every $v \in V$.

Proof. Let $v \in V$ and let $w \in W$ be such that $\|v-w\| \leq \varepsilon\|v\|$. Then

$$
\begin{aligned}
\|T v\| & =\|T v-T w+T w\| \\
& \geq\|T w\|-\|T(v-w)\| \\
& \geq C\|w\|-\varepsilon\|T\|\|v\| \\
& \geq C\|w-v+v\|-\varepsilon\|T\|\|v\| \\
& \geq C(\|v\|-\|v-w\|)-\varepsilon\|T\|\|v\| \\
& \geq(C-\varepsilon-\varepsilon\|T\|)\|v\| .
\end{aligned}
$$


Lemma 21. Let $S, T: E \rightarrow E^{\prime}$ be bounded linear operators between Banach spaces $E$ and $E^{\prime}$. Let $\|T-S\| \leq \varepsilon$ and assume that $S$ has closed range. Then there exists a constant $\delta=\delta(\varepsilon, S)$, such that $\operatorname{im} S$ is $\delta$-close to $\operatorname{im} T$ in $E^{\prime}$.

Proof. Let $w=S y \in \operatorname{im} S$. Since $S$ has closed image, there is a $C>0$ such that for every $S y$ there is $x \in E$ satisfying

$$
S y=S x
$$

and

$$
\|S x\| \geq C\|x\| .
$$

Consider $T x$. Then

$$
\|S x-T x\| \leq\|S-T\|\|x\| \leq \varepsilon C^{-1}\|S x\|
$$

Lemma 22. Let $S, T: E \rightarrow E^{\prime}$ be bounded linear operators between Banach spaces $E$ and $E^{\prime}$. Let $\|T-S\| \leq \varepsilon$ and assume that $S$ has closed range. Then there exists a constant $\delta=\delta(\varepsilon, S)$, such that $\operatorname{ker} T$ is $\delta$-close to $\operatorname{ker} S$.

Proof. Consider $x \in \operatorname{ker} T$. Then

$$
\|S x\|=\|S x-T x\| \leq \varepsilon\|x\| .
$$

Let $y \in$ be a vector satisfying

$$
d(0, \operatorname{ker} S+x)=\inf _{v \in \operatorname{ker} S}\|x+v\|=\|y\| .
$$

We have

$$
K\|y\| \leq\|S y\|,
$$

for some $K>0$, independent of $x$. Then

$$
x-y \in \operatorname{ker} S,
$$

and

$$
\|x-(x-y)\|=\|y\| \leq K^{-1}\|S y\|=K^{-1}\|S x\| \leq K^{-1} \varepsilon\|x\| .
$$

We will also be interested in comparing operators on quotients spaces of a Banach spaces. The following lemma will be crucial in this context.

Proposition 23. Let $V, W \subseteq E$ be closed subspaces of a Banach space $E$, where $V$ is $\varepsilon$-close to $W$. Let $w^{\prime}$ be such that $\left\|w^{\prime}\right\|_{E} \leq\left\|w^{\prime}+w\right\|_{E}$ for any $w \in W$ and consider the affine subspace $V+w^{\prime}$. Let $v^{\prime}$ be such that

$$
\left\|v^{\prime}\right\| \leq\left\|v^{\prime}+v\right\|
$$

for all $v \in V$ and

$$
v^{\prime}-w^{\prime} \in V
$$

Then

$$
\left\|v^{\prime}\right\| \geq(1-2 \varepsilon)\left\|w^{\prime}\right\|
$$


Proof. In general we have

$$
\left\|v^{\prime}-w^{\prime}\right\| \leq\left\|v^{\prime}\right\|+\left\|w^{\prime}\right\| \leq 2\left\|w^{\prime}\right\|,
$$

since by definition, $\left\|v^{\prime}\right\| \leq\left\|w^{\prime}\right\|$.

Now assume that there is some $v^{\prime}$ as above such that

$$
\left\|v^{\prime}\right\|<(1-2 \varepsilon)\left\|w^{\prime}\right\| \text {. }
$$

Since $V$ is $\varepsilon$-close to $W$, there exists $w \in W$ such that

$$
\left\|\left(v^{\prime}-w^{\prime}\right)-w\right\|=\left\|v^{\prime}-\left(w^{\prime}+w\right)\right\| \leq \varepsilon\left\|v^{\prime}-w^{\prime}\right\| .
$$

We have

$$
\begin{aligned}
\left\|w^{\prime}\right\| & \leq\left\|w+w^{\prime}\right\| \\
& \leq\left\|w+w^{\prime}-v^{\prime}\right\|+\left\|v^{\prime}\right\| \\
& <\varepsilon\left\|v^{\prime}-w^{\prime}\right\|+(1-2 \varepsilon)\left\|w^{\prime}\right\|
\end{aligned}
$$

From this it follows that

$$
\left\|w^{\prime}\right\|+(2 \varepsilon-1)\left\|w^{\prime}\right\|<\varepsilon\left\|v^{\prime}-w^{\prime}\right\|,
$$

so

$$
2\left\|w^{\prime}\right\|<\left\|v^{\prime}-w^{\prime}\right\|
$$

However, by (1),

$$
2\left\|w^{\prime}\right\|<\left\|v^{\prime}-w^{\prime}\right\| \leq 2\left\|w^{\prime}\right\|,
$$

which gives a contradiction.

For a subspace $V \subseteq X$ and $x \in X$ denote by $[x]_{V}$ the affine subspace $V+x$. The following statement gives a quantitative way of comparing operators on certain quotient spaces of Banach spaces.

Proposition 24. Let $V, W \subseteq E$ be two closed subspaces of a Banach space $E$. Assume that $V$ is $\varepsilon$-close to $W$. Let $T: E / V \rightarrow E^{\prime}$ and $S: E / W \rightarrow E^{\prime}$ be bounded linear operators, and let $\widetilde{T}, \widetilde{S}: E \rightarrow E^{\prime}$ denote their lifts to $E$. Assume that $\|\widetilde{T}-\widetilde{S}\| \leq \delta$. If $T$ is bounded below by $C$ then $S$ is bounded below by

$$
C(1-2 \varepsilon)-\delta \text {. }
$$

Proof. Let

$$
S\left([x]_{W}\right)=\widetilde{S} w^{\prime},
$$

where $w^{\prime}$ minimizes the distance to $[x]_{W}$. Then

$$
\begin{aligned}
\left\|\widetilde{S} w^{\prime}\right\| & \geq\left\|\widetilde{T} w^{\prime}\right\|-\left\|\widetilde{S} w^{\prime}-\widetilde{T} w^{\prime}\right\| \\
& \geq C\left\|v^{\prime}\right\|_{E}-\delta\left\|w^{\prime}\right\|_{E},
\end{aligned}
$$

where $w^{\prime}-v^{\prime} \in V$ and $v^{\prime}$ minimizes the distance to $\left[w^{\prime}\right]_{V}$.

Now, by the fact that $V$ is $\varepsilon$-close to $W$ and by the previous lemma we get

$$
\left\|\widetilde{S} w^{\prime}\right\| \geq C(1-2 \varepsilon)\left\|w^{\prime}\right\|_{E}-\delta\left\|w^{\prime}\right\|_{E},
$$

which means

$$
\left\|S\left[w^{\prime}\right]\right\| \geq(C(1-2 \varepsilon)-\delta)\left\|\left[w^{\prime}\right]\right\|_{X / W} .
$$




\section{Cohomology of Deformations}

We will now use the notion of close subspaces to study subspaces of Banach spaces related to cohomology. For a $(k-1)$-simplex $\tau$ denote by $\operatorname{ind}_{k}(\tau)$ the number of $k$-simplices $\sigma$ such that $\tau \subset \sigma$.

Lemma 25. Let $\pi$ be a representation of a finitely generated group $\Gamma$ on a Banach space $E$. Let $\rho$ be an $\varepsilon$-deformation of $\pi$. Then

$$
\left\|d_{\pi}^{n}-d_{\rho}^{n}\right\| \leq C(\Gamma, X, \pi, p, \varepsilon, n),
$$

as operators $C_{(p)}^{n}(X, \Gamma, E) \rightarrow C_{(p)}^{n+1}(X, \Gamma, E)$, where $C(\Gamma, X, \pi, p, \varepsilon, n) \rightarrow 0$ as $\varepsilon \rightarrow$ 0 .

Proof. Let $f \in C_{(p)}^{n}(X, E)$.

$$
\begin{aligned}
&\left\|d_{\pi}^{n} f-d_{\rho}^{n} f\right\|^{p}= \sum_{\sigma \in F^{(n)}}\left\|\sum_{i=0}^{n}(-1)^{i} \pi_{\gamma(\sigma)}^{-1} \pi_{\gamma\left(\sigma_{i}\right)} f\left(\sigma_{i}\right)-\sum_{i=0}^{n}(-1)^{i} \rho_{\gamma(\sigma)}^{-1} \rho_{\gamma\left(\sigma_{i}\right)} f\left(\sigma_{i}\right)\right\|^{p} \\
& \leq C^{\prime} \sum_{\sigma \in F^{(n)}}\left(\left\|\sum_{i=0}^{n}(-1)^{i}\left(\pi_{\gamma(\sigma)}^{-1}-\rho_{\gamma(\sigma)}^{-1}\right) \pi_{\gamma\left(\sigma_{i}\right)} f\left(\sigma_{i}\right)\right\|^{p}\right. \\
&\left.+\left\|\sum_{i=0}^{n}(-1)^{i}\left(\rho_{\gamma(\sigma)}^{-1} \pi_{\gamma\left(\sigma_{i}\right)}-\rho_{\gamma(\sigma)}^{-1} \rho_{\gamma\left(\sigma_{i}\right)}\right) f\left(\sigma_{i}\right)\right\|^{p}\right) \\
& \leq C^{\prime} \sum_{\sigma \in F^{(n)}}\left\|\pi_{\gamma(\sigma)^{-1}}-\rho_{\gamma(\sigma)^{-1}}\right\|^{p}\left\|\sum_{i=0}^{n}(-1)^{i} \pi_{\gamma\left(\sigma_{i}\right)} f\left(\sigma_{i}\right)\right\|^{p} \\
&+\left\|\rho_{\gamma(\sigma)}^{-1}\right\|^{p}\left\|\sum_{i=0}^{n}(-1)^{i}\left(\pi_{\gamma\left(\sigma_{i}\right)}-\rho_{\gamma\left(\sigma_{i}\right)}\right) f\left(\sigma_{i}\right)\right\|^{p}
\end{aligned}
$$

where $C^{\prime}$ depends on $n, \pi$ and $p$. We then have

$$
\begin{aligned}
\sum_{\sigma \in F^{(n)}}\left\|\sum_{i=0}^{n}(-1)^{i}\left(\pi_{\gamma\left(\sigma_{i}\right)}-\rho_{\gamma\left(\sigma_{i}\right)}\right) f\left(\sigma_{i}\right)\right\|^{p} & \leq C^{\prime \prime} \sum_{\sigma \in F^{(n)}} \sum_{i=0}^{n}\left\|\left(\pi_{\gamma\left(\sigma_{i}\right)}-\rho_{\gamma\left(\sigma_{i}\right)}\right) f\left(\sigma_{i}\right)\right\|^{p} \\
& \left.\leq C^{\prime \prime} n\left(\sup _{\sigma \in F^{(n)}} \| \pi_{\gamma\left(\sigma_{i}\right)}-\rho_{\gamma\left(\sigma_{i}\right)}\right) \|^{p}\right)\left(\sum_{\sigma_{i}}\left\|f\left(\sigma_{i}\right)\right\|^{p}\right) \\
& \leq C^{\prime \prime} n\left(\sup _{\tau(\mathrm{n}-1) \text {-simplex }} \operatorname{ind}_{k}(\tau)\right) \varepsilon\|f\|^{p} .
\end{aligned}
$$

Altogether, we obtain

$$
\begin{aligned}
\left\|d_{\pi}^{n} f-d_{\rho}^{n} f\right\| & \leq\left(\sup _{\tau(\mathrm{n}-1)-\operatorname{simplex}} \operatorname{ind}_{k}(\tau)\right) \varepsilon\|f\|+\varepsilon\left(\sup _{\sigma \in F^{(n)}}\left\|\rho_{\gamma(\sigma)}\right\|\right)\|f\| \\
& \leq\|f\| C(\Gamma, X, \pi, n, \varepsilon, p),
\end{aligned}
$$

since

$$
\left\|\rho_{\gamma}\right\| \leq\left\|\pi_{\gamma}\right\|+\varepsilon
$$


4.1. Deformations of cocycles. An important point that we would like to make is that the fact that the cohomology group $H^{n+1}(\Gamma, \pi)$ is reduced together with the finiteness condition $\mathrm{F}_{n+1}$, allows to deform $n$-cocycles of a deformation $\rho$ of $\pi$ to cocycles for $\pi$.

Theorem 26. Let $\Gamma$ be of type $F_{n+1}$ with a corresponding Eilenberg-MacLane space $X$ and let $\pi$ be a representation of $\Gamma$ on $E$. Let $\varepsilon>0$ and let $\rho$ be an $\varepsilon$-deformation of $\pi$. If the following two conditions hold:

(1) $H^{n}(\Gamma, \pi)=0$,

(2) $H^{n+1}(\Gamma, \pi)$ is reduced,

then there exists a constant $C=C(\Gamma, X, n, \pi, \varepsilon)$, such that $\operatorname{ker} d_{\pi}^{n}$ is $C$-close to $\operatorname{ker} d_{\rho}^{n}$.

Proof. We apply Lemmas 22 and 25 to $d_{\pi}^{n}$ and $d_{\rho}^{n}$. The constant $K$ appearing in the proof of lemma 22 in this setting is the $n$-Kazhdan constant $\kappa_{n}(\Gamma, X, p, \pi)$. Therefore $\operatorname{ker} d_{\rho}^{n}$ is $C$-close to $\operatorname{ker} d_{\pi}^{n}$, for an appropriate choice of the constant C.

In the case $n=1$ the above deformation theorem for cocycles has the following geometric interpretation. Given $\pi$ with $H^{2}(\Gamma, \pi)$ reduced, any affine action with linear part $\pi$ is close on the generators to an affine isometric action with linear part $\rho$.

For instance, in the case of the free group $\mathbb{F}_{n}$ the above principle applies to any deformation since free groups have cohomological dimension 1 and cohomology with any coefficients vanishes in degrees 2 and higher.

4.2. Proof of the main theorem. We are now in the position to prove the main result of the paper.

Theorem 27 (Theorem $1^{\prime}$ in the introduction). Let $\Gamma$ be a group acting on a uniformly locally finite simplicial complex $X, \pi$ be a representation of $\Gamma$ on a Banach space $E$ and $1 \leq p<\infty$. Assume that

(1) $L_{p} H^{n}(X, \Gamma, \pi)=0$,

(2) $L_{p} H^{n+1}(X, \Gamma, \pi)$ is reduced.

Then there exists a constant $\varepsilon=\varepsilon(\Gamma, X, \pi, n, p)>0$, such that for every $\varepsilon$-deformation $\rho$ of $\pi$ we have

$$
L_{p} H^{n}(X, \Gamma, \rho)=0 .
$$

Proof. We have the following diagrams, in which the top one is dual to the bottom one: 


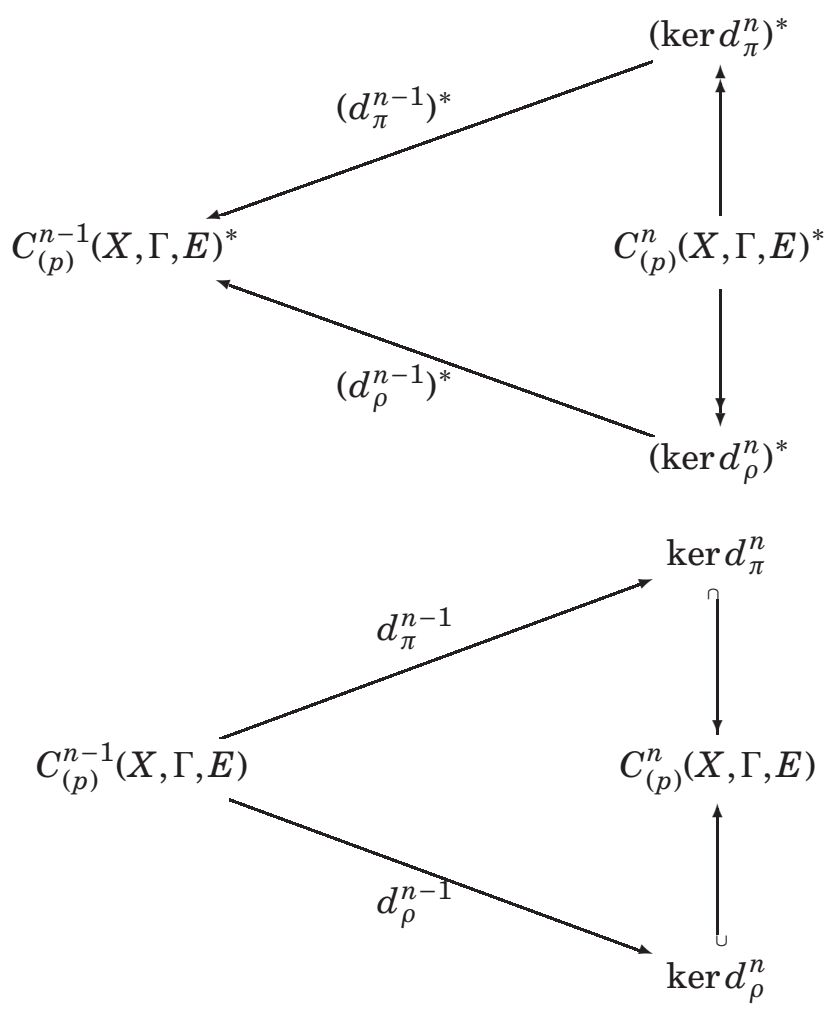

We need to show that $d_{\rho}^{*}$ is bounded below. Recall that the space $\left(\operatorname{ker} d_{\pi}\right)^{*}$ is isometrically isomorphic with the quotient space $C_{(p)}^{n}(X, \Gamma, E)^{*} / \operatorname{Ann}\left(\operatorname{ker} d_{\pi}\right)$. We need to consider several cases.

1) $d_{\pi}^{n-1}$ is the zero map (e.g., when $\left.n=0\right)$. Then $L_{p} H^{n}(X, \Gamma, \pi)=0$ if and only if

$$
\operatorname{ker} d_{\pi}^{n}=\operatorname{im} d_{\pi}^{n-1}=0 .
$$

If, additionally, im $d_{n}^{\pi}$ is closed then $d_{\pi}^{n}$ is bounded below and lemma 19 with $V=C_{(p)}^{n}(X, \Gamma, E)$ yields that $d_{\pi}^{n}$ is also bounded below. Consequently, ker $d_{\rho}^{n}=$ 0 , which implies $L_{p} H^{n}(X, \Gamma, \rho)=0$.

2) $d_{\pi}^{n}$ is the zero map. (This happens, e.g., when the dimension of the Eilenberg-MacLane space $X$ for the group $\Gamma$ is $n)$. In this case $\left(d_{\pi}^{n}\right)^{*}$ is bounded below on $C_{(p)}^{n}(X, \Gamma, E)^{*}$. By lemma 19, also $\left(d_{\rho}^{n}\right)^{*}$ is bounded below on $C_{(p)}^{n}(X, \Gamma, E)^{*}$.

3) $d_{\pi}^{n}$ is not zero. Since the range of $d_{\pi}^{n}$ is closed, we have

$$
\operatorname{Ann}\left(\operatorname{ker} d_{\pi}^{n}\right)=\operatorname{im}\left(d_{\pi}^{n}\right)^{*} .
$$

For $\rho$, on the other hand, we only have a dense inclusion $\operatorname{im}\left(d_{\rho}^{n}\right)^{*} \subseteq \operatorname{Ann}\left(\operatorname{ker} d_{\rho}^{n}\right)$. Since $\rho$ is an $\varepsilon$-deformation of $\pi$ and the range of $\left(d_{\pi}^{n}\right)^{*}$ is closed, there is a constant $\eta \geq 0$, given by lemma 21 , such that

$$
\operatorname{im}\left(d_{\pi}^{n}\right)^{*} \text { is } \eta \text {-close to } \operatorname{im}\left(d_{\rho}^{n}\right)^{*} .
$$


For the annihilators, this implies

$$
\operatorname{Ann}\left(\operatorname{ker} d_{\pi}^{n}\right)=\operatorname{im}\left(d_{\pi}^{n}\right)^{*} \quad \text { is } \eta \text {-close to } \operatorname{Ann}\left(\operatorname{ker} d_{\rho}^{n}\right)=\overline{\operatorname{im}\left(d_{\rho}^{n}\right)^{*}} .
$$

We can now compare the operators $\left(d_{\pi}^{n}\right)^{*}$ and $\left(d_{\rho}^{n}\right)^{*}$. Indeed, since $\left(d_{\pi}^{n}\right)^{*}$ is bounded below on $C_{(p)}^{n}(X, E)^{*} / \operatorname{Ann}\left(\operatorname{ker} d_{\pi}^{n}\right)$, then the above and lemmas 25 and 24 yield that for sufficiently small $\varepsilon \geq 0$, the operator $\left(d_{\rho}^{n}\right)^{*}$ is also bounded below on the quotient space $\left(\operatorname{ker} d_{\rho}^{n}\right)^{*}=C_{(p)}^{n}(X, \Gamma, E)^{*} / \operatorname{Ann}\left(\operatorname{ker} d_{\rho}^{n}\right)$.

Finally, to compare the higher Kazhdan constants recall that

$$
d_{\pi}^{n}: C_{(p)}^{n-1}(X, \Gamma, E) / \operatorname{ker} d_{\pi}^{n-1} \rightarrow \operatorname{ker} d_{\pi}^{n}
$$

is an isomorphism, where $\kappa_{n-1}(\Gamma, X, p, \pi)$ is the lower bound. Therefore its adjoint,

$$
\left(d_{\pi}^{n}\right)^{*}:\left(\operatorname{ker} d_{\pi}^{n}\right)^{*} \rightarrow\left(C_{(p)}^{n-1}(X, \Gamma, E) / \operatorname{ker} d_{\pi}^{n-1}\right)^{*},
$$

is also an isomorphism with the same lower bound. Proposition 24 allows to estimate the lower bound on the operator

$$
\left(d_{\rho}^{n}\right)^{*}:\left(\operatorname{ker} d_{\rho}^{n}\right)^{*} \rightarrow\left(C_{(p)}^{n-1}(X) / \operatorname{ker} d_{\rho}^{n-1}\right)^{*},
$$

which we now know is an isomorphism, and the Kazhdan constant satisfies

$$
\kappa_{n-1}(\Gamma, X, p, \rho) \geq \kappa_{n-1}(\Gamma, X, p, \pi)-c(\Gamma, X, \pi, n, \varepsilon, p) .
$$

4.3. Proof in the Hilbert case. The following proof of Theorem 1 is specific to representations on Hilbert spaces and uses the properties of the Laplacian. It was provided to us by Roman Sauer.

Given a complex of Hilbert spaces,

$$
\ldots \longrightarrow C_{n-1} \stackrel{d^{n-1}}{\longrightarrow} C_{n} \stackrel{d_{n}}{\longrightarrow} C_{n+1} \longrightarrow \ldots
$$

consider the Laplacian,

$$
\Delta^{n}=d^{n-1}\left(d^{n-1}\right)^{*}+\left(d^{n}\right)^{*}\left(d^{n}\right): C_{n} \rightarrow C_{n}
$$

and recall that $\Delta^{n}$ is a chain map. Additionally, $d^{*}$ is a chain homotopy between $\Delta^{n}$ and 0 , by definition of $\Delta^{n}$. These two facts imply that there is an induced map $\bar{\Delta}^{n}$ on the cohomology $H^{n}$ of the above chain complex, and that $\bar{\Delta}^{n}=0$.

Theorem 28. Assume that $H^{n+1}$ is reduced. Then $H^{n}=0$ if and only if $\Delta^{n}$ is invertible.

Proof. Assume that $\Delta^{n}$ is invertible. Without loss of generality we can assume that $H^{n+1}=0$, by considering the chain complex truncated to im $d^{n}$, which is a closed subspace of $C^{n+1}$, by assumption. Let $x \in C^{n}$ be such that $d^{n} x=0$ and take $y \in C^{n}$ satisfying $\Delta^{n} y=x$. We have

$$
\Delta^{n} d^{n} y=d^{n} \Delta^{n} y=0,
$$

since $\Delta^{n}$ is a chain map. Since $\operatorname{ker} \Delta^{n}=0$, we conclude that $d^{n} y=0$. 
Now, since $\bar{H}^{n}=0$, we can choose a sequence $\left\{z_{i}\right\}$, satisfying

$$
d^{n} z_{i} \rightarrow y \text {. }
$$

Observe that

$$
d^{n}\left(d^{n}\right)^{*} d^{n} z_{i} \rightarrow d^{n}\left(d^{n}\right)^{*} y
$$

However, on the other hand we have

$$
\Delta^{n} d^{n} z_{i}=d^{n} \Delta^{n} z_{i}=d^{n}\left(d^{n}\right)^{*} d^{n} z_{i}+d^{n} d^{n-1}\left(d^{n-1}\right)^{*} z_{i} .
$$

The last term vanishes and we obtain

$$
d^{n}\left(d^{n}\right)^{*} d^{n} z_{i} \rightarrow \Delta^{n} y=x .
$$

It follows that

$$
x=d^{n}\left(\left(d^{n}\right)^{*} y\right),
$$

which implies vanishing of $H^{n}$.

To prove the converse assume $H^{n}=0$ and argue to show that $\Delta^{n}$ is invertible. Denote $Z^{n}=\operatorname{ker} d^{n}$ and $B^{n}=\operatorname{im} d_{n-1}$. Assume that $H^{n+1}$ is reduced and that $H^{n}=0$. This means that $B^{n+1}$ is closed in $C^{n+1}$ and that $B^{n}=Z^{n}$. In particular, this implies that $B^{n}$ is closed.

Consider the decompositions,

$$
\begin{aligned}
C^{n-1} & =Z^{n-1} \oplus\left(Z^{n-1}\right)^{\perp}, \\
C^{n} & =Z^{n} \oplus\left(Z^{n}\right)^{\perp}=B^{n} \oplus\left(B^{n}\right)^{\perp}, \\
C^{n+1} & =B^{n+1} \oplus\left(B^{n+1}\right)^{\perp} .
\end{aligned}
$$

We have that $\left(d^{n}\right)^{*} d^{n}$ restricted to $Z^{n}$ is the zero map. We claim that $\left(d^{n}\right)^{*} d^{n}$ is invertible on $\left(Z^{n}\right)^{\perp}$. Indeed, by the Open Mapping Theorem,

$$
d^{n}:\left(Z^{n}\right)^{\perp} \rightarrow B^{n+1}
$$

is an isomorphism. Since $\left(d^{n}\right)^{*}$ restricted to $B^{n+1}{ }^{\perp}$ is 0 , it follows that $\left(d^{n}\right)^{*}$ : $B^{n+1} \rightarrow\left(Z^{n}\right)^{\perp}$ is an isomorphism.

Now observe that $\left(d^{n-1}\right)\left(d^{n-1}\right)^{*}=0$ on $\left(B^{n}\right)^{\perp}$. We claim that $\left(d^{n-1}\right)\left(d^{n-1}\right)^{*}$ restricted to $B^{n}$ is invertible. Indeed, again by the Open Mapping Theorem, $d^{n-1}:\left(Z^{n-1}\right)^{\perp} \rightarrow B^{n}$ is an isomorphism and it follows that $\left(d^{n-1}\right)^{*}: B^{n} \rightarrow$ $\left(Z^{n-1}\right)^{\perp}$ is an isomorphism. It follows that $\Delta^{n}$ is invertible.

Applying the above argument to the cochain complex $C_{(2)}^{n}(X, \Gamma, E)$ with a given representation $\pi$ yields the assertion.

4.4. Assumptions on $H^{n+1}$ are essential. We will now show that the assumption that $H^{n+1}(\Gamma, \pi)$ being reduced is essential.

We will show this in degree 0 where cohomology is simply the subspace of invariant vectors of the representation. The examples we have in mind arise as representations without non-zero invariant vectors, but with almost invariant vectors. However, we additionally want the almost invariant vectors to arise as invariant vectors for deformations. An explicit example of this type is exhibited below. 
Consider the infinite cyclic group $\mathbb{Z}$. This group acts on $L_{2}\left(S^{1}\right)$, where the circle $S^{1}$ is viewed as the Pontraygin dual of $\mathbb{Z}$. The representation of $\mathbb{Z}$ on $L_{2}\left(S^{1}\right)$ is given by specifying the generator:

$$
T f(z)=e^{i z} f(z) .
$$

This representation does not have non-zero invariant vectors; that is, $H^{0}(\Gamma, \pi)=$ 0 .

We will now show that for every $\varepsilon>0$ there is an $\varepsilon$-deformation $\pi_{\varepsilon}$ of $\pi$ for which $H^{0}\left(\Gamma, \pi_{\varepsilon}\right)$ does not vanish. Choose a neighborhood $U$ of positive but sufficiently small measure around the identity in $S^{1}$ and define

$$
T_{\varepsilon} f(z)= \begin{cases}T f(z) & \text { if } z \notin U, \\ f(z) & \text { if } z \in U .\end{cases}
$$

Choosing the neighborhood $U$ to have sufficiently small measure we ensure that

$$
\left\|T-T_{\varepsilon}\right\| \leq \varepsilon,
$$

which means that the representation $\pi_{\varepsilon}$ generated by $T_{\varepsilon}$ is an $\varepsilon$-deformation of $\pi$. On the other hand, $\pi_{\varepsilon}$ has non-zero invariant vectors, as long as $U$ has positive measure. That is, $H^{0}\left(\mathbb{Z}, \pi_{\varepsilon}\right) \neq 0$, as claimed.

Note, that $H^{1}(\mathbb{Z}, \pi)$ is not reduced. Indeed, if $\rho$ is an $\varepsilon$-deformation of $\pi$ and $\rho$ has an invariant vector $v$, then $v$ is $\varepsilon$-invariant for $\pi$, in the sense that

$$
\left\|\pi_{s} v-v\right\| \leq \varepsilon \text {. }
$$

Thus $\pi$ has a sequence of almost invariant vectors but no non-zero invariant vectors, i.e., the range of $d_{\pi}^{0}$ is not closed.

\section{FinAL REMARKS}

5.1. Higher property $(\mathbf{T})$ and reduced cohomology. By a cohomological characterization of property $(\mathrm{T})$ one usually means a theorem of Delorme and Guichardet: $\Gamma$ has property $(\mathrm{T})$ if and only if $H^{1}(\Gamma, \pi)=0$ for every unitary representation $\pi$. However, there is another way to formulate property (T) in terms of cohomology, that fits very well with our setup.

Proposition 29. The following conditions are equivalent for a finitely generated group:

(1) $\Gamma$ has property (T);

(2) for every unitary representation $\pi, H^{1}(\Gamma, \pi)$ is reduced.

Indeed, $H^{0}(\Gamma, \pi)$ consists of the $\pi$ invariant vectors on the representation space and the image of the first codifferential is closed precisely when there are no almost invariant vectors. Thus the above proposition is a cohomological reformulation of Kazhdan's original definition. The Delorme-Guichardet theorem states that $H^{1}(\Gamma, \pi)$ is reduced for every unitary $\pi$ if and only if it vanishes for every $\pi$.

A generalization property ( $\mathrm{T}$ ) to higher dimensions can now be done in two ways. The first one amounts to requesting that $H^{n}(\Gamma, \pi)$ vanishes for every 
unitary representation. Examples of groups satisfying such conditions for $n \geq 2$ were discussed in $[4,9,17]$. The second route is via the following

Definition 30. Let $\Gamma$ be a group of type $F_{n+1}$. We say that $\Gamma$ has property $\left(T_{n}\right)$ if for every unitary representation $\pi, H^{n+1}(\Gamma, \pi)$ is reduced.

Kazhdan's property (T) is then property $\left(\mathrm{T}_{0}\right)$. It is clear that, similarly as in the classical case, the vanishing of $H^{n+1}(\Gamma, \pi)$ for every unitary $\pi$ trivially implies property $\left(T_{n}\right)$. We do not know if the converse is true. If the answer is positive, such an equivalence would provide a higher-dimensional analog of the Delorme-Guichardet theorem.

Interestingly, groups satisfying the conditions of Definition 30 fit perfectly into our framework and for these groups the conditions of Theorem 1 are automatically satisfied.

5.2. Weil's local rigidity criterion. Let $\Gamma$ be a finitely generated group with a generating set $S$ and let $G$ be a Lie group. Consider the set of homomorphisms, $\operatorname{Hom}(\Gamma, G)$. A homomorphism $\varphi \in \operatorname{Hom}(\Gamma, G)$ is said to be locally rigid if there exists $\varepsilon>0$ such that for any homomorphism $\psi \in \operatorname{Hom}(\Gamma, G)$ satisfying

$$
\sup _{s \in S} d_{G}(\varphi(s), \psi(s)) \leq \varepsilon,
$$

there exists $g \in G$ such that

$$
\psi(\gamma)=g^{-1} \varphi(\gamma) g .
$$

Weil $[21,22]$ proved the following criterion for local rigidity.

Theorem 31 (A. Weil). For $\varphi$ as above, if $H^{1}(\Gamma, \operatorname{Ad} \circ \varphi)=0$ then $\varphi$ is locally rigid.

Now we will apply Theorem 1 . Consider a homomorphism $\varphi \in \operatorname{Hom}(\Gamma, G)$ and assume that the condition in Weil's theorem is satisfied for $\varphi: H^{1}(\Gamma, \operatorname{Ad} \circ \varphi)=$ 0 . Note that the representation $\operatorname{Ad} \circ \varphi$ is a representation on the vector space underlying the Lie algebra $\mathfrak{G}$. Since the $G$ is finite dimensional, so is $\mathfrak{G}$ and it follows that $H^{i}(\Gamma, \operatorname{Ad} \circ \varphi)$ is reduced for all $i$. Therefore, by Theorem 1 , there exists a $\varepsilon_{\varphi}>0$, such that for any $\psi \in \operatorname{Hom}(\Gamma, G)$, satisfying

$$
\sup _{s \in S} d_{G}(\varphi(s), \psi(s)) \leq \varepsilon_{\varphi}
$$

we have $H^{1}(\Gamma, \operatorname{Ad} \circ \psi)=0$.

Theorem 32. Let $\Gamma, G$ be as above, where $\Gamma$ is finitely presented. The set

$$
W=\left\{\varphi \in \operatorname{Hom}(\Gamma, G): H^{1}(\Gamma, \operatorname{Ad} \circ \varphi)=0\right\}
$$

is open in the uniform topology. 
5.3. Vanishing vs connectedness of $\operatorname{Hom}(\Gamma, U(\not{H}))$. Consider now $\operatorname{Hom}(\Gamma, U(\not{H}))$, the space of homomorphisms from $\Gamma$ into the unitary group of a Hilbert space $\mathscr{H}$. Theorem 1 yields the following consequence about the metric and topological structure of $\operatorname{Hom}(\Gamma, U(\not{H}))$.

Throughout this section we assume that $\Gamma$ is a group of type $F_{n}$ and cohomological dimension $n$. We have a natural decomposition

$$
\operatorname{Hom}(\Gamma, U(\mathscr{H}))=N_{\Gamma, \mathscr{H}} \sqcup V_{\Gamma, \mathscr{H}},
$$

where

$$
V_{\Gamma, \mathscr{H}}=\left\{\pi \in \operatorname{Hom}(\Gamma, U(\mathscr{H})): H^{n}(\Gamma, \pi)=0\right\},
$$

and

$$
N_{\Gamma, \mathscr{H}}=\left\{\pi \in \operatorname{Hom}(\Gamma, U(\mathscr{H})): H^{n}(\Gamma, \pi) \neq 0\right\} .
$$

Proposition 33. The set $N_{\Gamma, \mathscr{H}}$ is closed.

Proof. Let $\pi_{n}$ be a sequence of elements of $N_{\Gamma, \mathscr{H}}$ converging to $\pi \in \operatorname{Hom}(\Gamma, U(\not{H}))$. If $H^{n}(\Gamma, \pi)=0$ then, by Theorem 1 , we would have $H^{n}\left(\Gamma, \pi_{n}\right)=0$ for $\pi_{n}$ for $n$ sufficiently large.

The set $V_{\Gamma, \mathscr{H}}$ is consequently open. However, under certain conditions $V_{\Gamma, \mathcal{H}}$ is also open. Let

$$
\kappa\left(S, V_{\Gamma, \mathscr{C}}\right)=\inf _{\pi \in V_{\Gamma, \mathscr{H}}} \kappa_{n}(\Gamma, S, \pi) .
$$

Proposition 34. If $\kappa\left(S, V_{\Gamma, \mathscr{H}}\right)>0$ then $V_{\Gamma, \mathcal{H}}$ is closed.

Proof. Let $\pi_{n} \in V_{\Gamma, \mathcal{H}}$ and $\pi_{n} \rightarrow \pi$. By the previous estimate, if $\rho$ is a $\varepsilon$ deformation of $\pi_{n}$ for

$$
\varepsilon<\frac{\kappa_{n-1}(\Gamma, S, \pi)}{K_{n-1}}
$$

then $H^{n}(\Gamma, \rho)=0$. Since the constant $K_{n-1}$ is independent of $\pi_{n}$, by the assumption on $\kappa\left(S, V_{\Gamma, \mathscr{H}}\right)$ we conclude that there exists $n$ sufficiently large such that $\pi$ is an $\varepsilon$-deformation for

$$
\varepsilon<\frac{\kappa\left(S, V_{\Gamma, \not{H}}\right)}{K_{n-1}} .
$$

Recall that the Delorme-Guichardet theorem states that if the Kazhdan constant of $\Gamma$ is positive then $H^{1}(\Gamma, \pi)=0$ for every unitary representation $\pi$. The following corollary can be regarded as a top-dimensional relative of the Delorme-Guichardet theorem.

Theorem 35. Let $\Gamma$ be such that $\kappa\left(S, V_{\Gamma, U(\not \mathcal{C})}\right)>0$. Then vanishing of $H^{n}(\Gamma, \pi)$ is stable on connected components of $\operatorname{Hom}(\Gamma, U(\mathscr{H}))$.

More precisely, let $\mathscr{C} \subseteq \operatorname{Hom}(\Gamma, U(\mathcal{H}))$ be a connected component. The following conditions are equivalent:

(1) $H^{n}(\Gamma, \pi)$ vanishes for some $\pi \in \mathscr{C}$.

(2) $H^{n}(\Gamma, \pi)$ vanishes for all $\pi \in \mathscr{C}$. 


\section{REFERENCES}

[1] T. Austin, Euclidean-valued group cohomology is always reduced, arXiv:1310.3210 [math.GR]. Preprint, 2013.

[2] U. Bader, A. Furman, T. Gelander, and N. Monod, Property (T) and rigidity for actions on Banach spaces, Acta Math. 198 (2007), no. 1, 57-105.

[3] U. Bader, T. Gelander, and N. Monod, A fixed point theorem for $L^{1}$ spaces, Invent. Math. 189 (2012), no. 1, 143-148.

[4] W. Ballmann and J. Świątkowski, On $L^{2}$-cohomology and property (T) for automorphism groups of polyhedral cell complexes, Geom. Funct. Anal. 7 (1997), no. 4, 615-645.

[5] B. Bekka, P. de la Harpe, and A. Valette, Kazhdan's property (T), New Mathematical Monographs, vol. 11, Cambridge University Press, Cambridge, 2008.

[6] A. Borel and N. Wallach, Continuous cohomology, discrete subgroups, and representations of reductive groups, 2nd ed., Mathematical Surveys and Monographs, vol. 67, American Mathematical Society, Providence, RI, 2000.

[7] K. S. Brown, Cohomology of groups, Graduate Texts in Mathematics, vol. 87, SpringerVerlag, New York, 1994. Corrected reprint of the 1982 original.

[8] M. Burger, N. Ozawa, and A. Thom, On Ulam stability, Israel J. Math. 193 (2013), no. 1, $109-129$

[9] J. Dymara and T. Januszkiewicz, Cohomology of buildings and their automorphism groups, Invent. Math. 150 (2002), no. 3, 579-627.

[10] M. Ershov and A. Jaikin-Zapirain, Property (T) for noncommutative universal lattices, Invent. Math. 179 (2010), no. 2, 303-347.

[11] D. Fisher and G. Margulis, Almost isometric actions, property (T), and local rigidity, Invent. Math. 162 (2005), no. 1, 19-80.

[12] R. Geoghegan, Topological methods in group theory, Graduate Texts in Mathematics, vol. 243, Springer, New York, 2008.

[13] S. Heinrich and P. Mankiewicz, Applications of ultrapowers to the uniform and Lipschitz classification of Banach spaces, Studia Math. 73 (1982), no. 3, 225-251.

[14] J. Koivisto, Automorphism groups of simplicial complexes and rigidity for uniformly bounded representations, Geom. Dedicata 169 (2014), 57-82.

[15] A. Lubotzky, What is...property ( $\tau$ )?, Notices Amer. Math. Soc. 52 (2005), no. 6, 626-627.

[16] M. Mimura, Fixed point properties and second bounded cohomology of universal lattices on Banach spaces, J. Reine Angew. Math. 653 (2011), 115-134.

[17] I. Oppenheim, Vanishing of cohomology and property (T) for groups acting on weighted simplicial complexes, arXiv:1110.5724 [math.GR].

[18] P. W. Nowak, Poincaré inequalities and rigidity for actions on Banach spaces, Journal of the European Mathematical Society (JEMS), (to appear).

[19] P. W. Nowak, Group actions on Banach spaces, Handbook of group actions (L. Ji, A. Papadopoulos and S.-T. Yau, ed.) Higher Education Press and International Press (to appear).

[20] G. Pisier, Similarity problems and completely bounded maps, Second, expanded edition, Lecture Notes in Mathematics, vol. 1618, Springer-Verlag, Berlin, 2001. Includes the solution to "The Halmos problem".

[21] A. Weil, On discrete subgroups of Lie groups, Ann. of Math. (2) 72 (1960), 369-384.

[22] A. Weil, On discrete subgroups of Lie groups. II, Ann. of Math. (2) 75 (1962), 578-602.

TECHNION, HAIFA, ISRAEL

E-mail address: uri.bader@gmail.com

Instytut Matematyczny Polskiej Akademii Nauk, Warsaw, Poland

Instytut Matematyki, Uniwersytet Warszawski, WarsaW, Poland

E-mail address: pnowak@mimuw.edu.pl 